INDO GLOBAL JOURNAL OF

PHARMACEUTICAL SCIENCES

ISSN 2249- 1023

\title{
Gut Microflora in Human Health and Disease
}

\author{
Nitin Sharma; Gauri Khanna, Nistha Saxena; Prakriti Shome, Kopal Singhal, Sujata Mohanty * \\ Department of Biotechnology, Jaypee Institute of Information Technology, A-10, Sector 62, Noida, 201309, India
}

Address for Correspondance: Sujata Mohanty, sujata.mohanty@jiit.ac.in

Keywords Gut

Microflora; Disease

Prevention;

Probiotics; Human

Health.

\begin{abstract}
Human beings harbour at least 100 trillion microbial cells in and on the human body which constitute our internal and external microbiota and the genes these cells encode for, known as our microbiome. The microbiota is referred to as "forgotten organ" and plays a major role in human health and disease etiology. Several factors such as age, genetics, environment, diet, antibiotic, surgery, drug treatments or transplantations play a key role in establishing microbiota population in the host. The gut microbiota offers several benefit to the host by maintaining optimum physiological conditions inside the gut, thereby providing resistance from pathogens, extracting energy and nutrients from diets and contributing in immune system functioning and development. Literature suggest an inverse relation between autoimmune diseases (Inflammatory bowel disease, psoriasis, rheumatoid arthritis) and the microbiota population. The concept that some bacteria can provide a health benefit and cure disease, led the way to the development of probiotics which can be defined as 'live microorganisms that, when administered in adequate amounts, confer a health benefits on the host'. The major groups of probiotic bacteria used as human foods supplements for treatment of lactose intolerance in adults are lactobacilli and streptococci. Also the bacterium Oxalobacter formigenes is able to reduce the risk of developing kidney stones by degrading oxalate in the lumen of the intestine. Another multifactorial mechanism of action of some probiotics is to improve human health by influencing the resident microbiota and exclusion of exogenous microbes e.g. certain probiotic strains can secrete antibiotic-like factors to prevent localized growth of potential competitors thereby preventing the growth of exogenous microbes and thus protecting the organism from developing diarrhea. Gut microflora thus play a significant role in affecting the host susceptibility to diseases and overtime also leads to epigenetic changes in the gut microbiome. An understanding of the important functional roles of these microbiota in human gut will prove instrumental in medical biology and disease pathogenesis. (C) 2016 iGlobal Research and Publishing Foundation. All rights reserved.
\end{abstract}

Conference Proceedings: International Conference on Advances in Plant and Microbial Biotechnology (PMB2017); JIIT, Noida: February 02-04, 2017

Indo Global Journal of Pharmaceutical Sciences( ISSN 22491023 ; CODEN- IGJPAI; NLM ID: 101610675) indexed and abstracted in EMBASE(Elsevier), SCIRUS(Elsevier),CABI, CAB Abstracts, Chemical Abstract Services(CAS), American Chemical Society(ACS), Index Copernicus, EBSCO, DOAJ, Google Scholar and many more. For further details, visit http://iglobaljournal.com 